\title{
APLICAÇÃO DO MODELO DO NÚCLEO NÃO REAGIDO À LIXIVIAÇÃO DA CALCOPIRITA PELO ÍON Fe ${ }^{+3}$
}

Rodrigo Rangel Porcaro Flávio Luiz Martins ${ }^{2}$ Versiane Albis Leão ${ }^{3}$

\section{Resumo}

A lixiviação da calcopirita pelo íon férrico é um processo considerado lento e com baixa recuperação de cobre, fato atribuído à passivação do mineral. Nesse sentido, o presente trabalho investiga a cinética de lixiviação de uma amostra de calcopirita de elevada pureza, utilizando sulfato férrico como oxidante. Os efeitos da agitação, temperatura, Eh e concentração de $\mathrm{Fe}^{3+}$ na extração de cobre foram avaliados. Os dados experimentais seguiram o modelo do núcleo não-reagido com controle por difusão na camada de cinzas e alta energia de ativação $(103,9 \pm 6,5 \mathrm{~kJ} / \mathrm{mol})$, provavelmente devido à não consideração do efeito da distribuição de tamanhos de partículas (PSD) nas equações cinéticas. A aplicação da hipótese do estado quasi-estacionário em sistemas líquido-sólido bem como o efeito da PSD na interpretação dos dados cinéticos são discutidos.

Palavras-chave: Calcopirita; Modelo do núcleo não-reagido; Energia de ativação.

\section{MODELLING CHALCOPYRITE LEACHING BY Fe ${ }^{+3}$ IONS WITH THE SHRINKING CORE MODEL}

\begin{abstract}
Chalcopyrite leaching by ferric iron is considered a slow process with low copper recovery; a phenomenon ascribed to the passivation of the mineral surface during leaching. Thus, the current study investigated the leaching kinetics of a high purity chalcopyrite sample in the presence of ferric sulfate as oxidant. The effects of the stirring rate, temperature, Eh and $\mathrm{Fe}^{3+}$ concentration on copper extraction were assessed. The leaching data could be described by the shirking core model (SCM) for particles of unchanging size and indicated diffusion in the ash layer as the rate-controlling step with a high activation energy $(103.9 \pm 6.5 \mathrm{~kJ} / \mathrm{mol})$; likely an outcome of neglecting the effect of particle size distribution (PSD) on the kinetics equations. Both the application of the quasi-steady-state assumption to solid-liquid systems and the effect of the particle size distribution on the interpretation of kinetics data are also discussed.
\end{abstract}

Keywords: Chalcopyrite; Shrinking core model; Activation energy.

\section{INTRODUÇÃO}

A Hidrometalurgia é o campo da metalurgia extrativa que utiliza soluções aquosas para extração de metais. As aplicações tradicionais envolvem a metalurgia do ouro, urânio, zinco, cobre, níquel, titânio, dentre outros, normalmente associada a sulfetos desses metais [I]. Seu grande campo de atuação é o processamento de minérios e rejeitos, particularmente quando o nível de impurezas deletérias como cloro, arsênio e flúor é elevado o que resulta em restrições ao tratamento pirometalúrgico de concentrados produzidos com tais minérios.
A lixiviação é a principal operação unitária dos processos hidrometalúrgicos e consiste na solubilização seletiva do metal de interesse. Dentre os reagentes, destacam-se os ácidos (como o sulfúrico), as bases (como o hidróxido de sódio), agentes complexantes (como o cianeto de sódio) e os oxidantes (oxigênio e ferro férrico). Utilizam-se condições variadas de temperatura e pressão para a etapa de lixiviação, usualmente de $25^{\circ} \mathrm{C}$ a $250^{\circ} \mathrm{C}$. A lixiviação pode ser intermediada por micro-organismos (biolixiviação), de grande aplicação na dissolução de sulfetos [2].

'Instituto Federal de Minas Gerais - IFMG, Campus Ouro Branco, Ouro Branco, MG, Brasil.

${ }^{2}$ Rede Temática em Engenharia de Materiais - REDEMAT, Universidade Federal de Ouro Preto - UFOP, Ouro Preto, MG, Brasil.

${ }^{3}$ Laboratório de Bio\&Hidrometalurgia, Departamento de Engenharia Metalúrgica e de Materiais, Universidade Federal de Ouro Preto - UFOP, Campus Morro do Cruzeiro, Ouro Preto, MG, Brasil.E-mail: versiane@demet.em.ufop.br; versiane.ufop@gmail.com 
Um dos sulfetos cuja lixiviação é muito estudada é a calcopirita $\left(\mathrm{CuFeS}_{2}\right)$. Este é o principal sulfeto do cobre e requer a presença de um agente oxidante para sua dissolução, normalmente soluções de sulfato ou cloreto férrico, além de oxigênio. Entretanto, a calcopirita apresenta refratariedade aos processos de solubilização em pressão ambiente e na presença de íons férricos o que é atribuída à passivação de sua superfície [3]. Hackl et al. [4] sugerem que a passivação é controlada pela formação e evolução de uma camada de polissulfeto rica em cobre e menos reativa do que a calcopirita, enquanto Cordoba et al. [5] atribuem a passivação à formação de uma camada superficial de jarosita.

Indepentendemente da natureza da passivação, a dissolução da calcopirita é incompleta e tem cinética lenta. Dessa forma vários estudos cinéticos têm sido conduzidos objetivando determinar a etapa controladora do processo de lixiviação. Dentre os modelos desenvolvidos para reações fluido-sólido não catalíticas, podem ser citados: (i) o modelo do núcleo não reagido (shrinking core model, SCM) para partículas de tamanho constante, (ii) o SCM para partículas que diminuem de tamanho (SPM) e (iii) o modelo do grão [6]. Como exemplo, o SCM foi aplicado para modelar a cinética de lixiviação da esfalerita (ZnS) [7]; o SPM foi utilizado na dissolução alcalina de minérios oxidados de zinco [8] enquanto a lixiviação de silicatos de zinco foi modelada utilizando o modelo do grão [9]. Nesse trabalho, algumas premissas do modelo do núcleo não reagido quando aplicado a reações sólido-líquido, bem como o efeito da distribuição de tamanho nas equações cinéticas são revistos e aplicados à dissolução da calcopirita pelo íon férrico.

\section{MATERIAIS E MÉTODOS}

\section{I Caracterização da Amostra de Calcopirita}

Foi utilizada amostra de mão com elevado teor de calcopirita. A amostra foi inicialmente homogeneizada e quarteada, utilizando-se o método de pilhas longitudinais. Uma amostra foi reservada para determinação dos teores de cobre e ferro (em A. A. Perkin Elmer, modelo AAnalyst 100) e enxofre (ICP-OES, Agilent, modelo 725), através de dissolução em água régia $\left(3 \mathrm{HCl}-\mathrm{IHNO}_{3}, \mathrm{v} / \mathrm{v}\right)$, sendo encontrados teores de $32.9 \% \mathrm{Cu}, 25.9 \%$ Fe e $34.0 \% \mathrm{~S}$. A presença de calcopirita na amostra foi confirmada por difração de raios- $x$ e o conteúdo do mineral foi estimado em $95 \%$.

Para os ensaios de lixiviação, o sulfeto de cobre foi pulverizado a seco em moinho vibratório de discos, seguida de lavagem com água ácida $(50 \% \mathrm{HCl}, \mathrm{v} / \mathrm{v})$ para remoção de filmes de óxidos formados devido à cominuição. A lavagem ácida durou 2 minutos, seguida de lavagem com água destilada. As amostras foram então secas em temperatura ambiente, separadas em alíquotas de $5 \mathrm{~g}$ e estocadas em freezer para reduzir a oxidação superficial. A distribuição granulométrica, Figura I, da amostra lavada foi determinada em um granulômetro Cilas, modelo 1064 e indicou que $80 \%$ da amostra era menor que $20 \mu \mathrm{m} \mathrm{e} \mathrm{o}_{50}$ igual era de $9,5 \mu \mathrm{m}$.

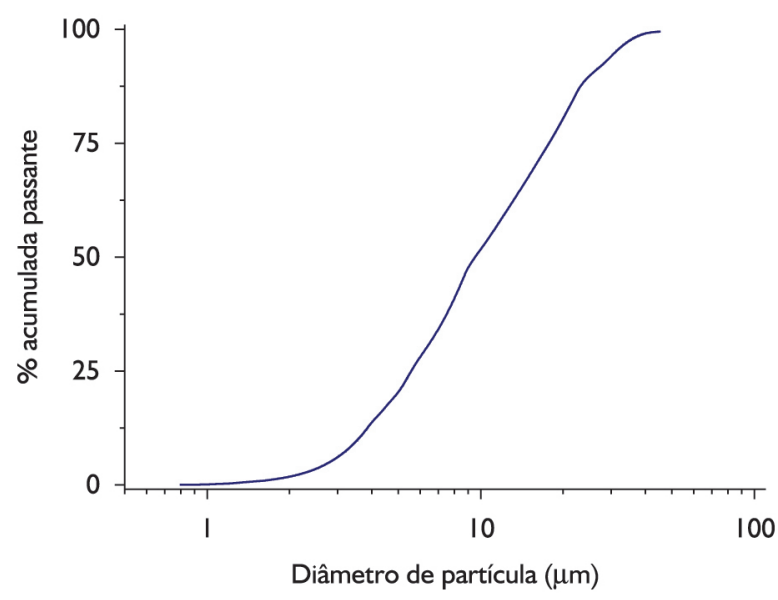

Figura I. Distribuição granulométrica da amostra de calcopirita pulverizada.

\subsection{Ensaios de Lixiviação}

Os ensaios de lixiviação foram realizados em batelada, utilizando um reator encamisado de vidro (Shott), com volume de $500 \mathrm{~mL}$. A temperatura do sistema era controlada por meio de circulação de água, com auxílio de um banho termostatizado com bomba de circulação externa (Nova Ética). Amostras de $3 \mathrm{~mL}$ da fase líquida eram recolhidas em intervalo regular de tempo para a determinação da concentração de cobre por ICP-OES.

A condição referência adota para realização dos ensaios foi: concentração de $\mathrm{Fe}^{+3}$ de $0,5 \mathrm{~mol} / \mathrm{L}$ (sulfato férrico p.a.), I mol/L de ácido sulfúrico, $90^{\circ} \mathrm{C}$ e agitação de $600 \mathrm{~min}^{-1}$; a concentração de sólidos adotada era igual a $0,1 \%$. A temperatura foi variada no intervalo de $50^{\circ} \mathrm{C}$ a $90^{\circ} \mathrm{C}$; a concentração de $\mathrm{Fe}^{+3}$ foi estudada no intervalo de 0,1 a $I, 5 \mathrm{~mol} / \mathrm{L}$. Durante a análise cinética, o efeito da agitação foi investigado no intervalo de $360 \mathrm{~min}^{-1}$ a $720 \mathrm{~min}^{-1}$, e observou-se que a partir e $600 \mathrm{~min}^{-1}$, a dissolução do cobre não era mais influenciada pela agitação.

As medidas de Eh foram realizadas em equipamento Eh-metro da Digimed, modelo DM20 com eletrodo de Ag/ $\mathrm{AgCl}-\mathrm{Pt}$. As soluções foram preparadas a partir de reagentes p.a. e água destilada, momentos antes da realização dos ensaios. Ao final dos mesmos, os resíduos da lixiviação foram filtrados, lavados com água destilada, secos em temperatura ambiente e observados em um MEV-EDS (JEOL JSM 50I).

\section{RESULTADOS E DISCUSSÃO}

Considerando que a lixiviação da calcopirita pelo íon $\mathrm{Fe}^{3+}$ pode ser representada pela Equação I, o efeito da temperatura e da concentração do oxidante na sua dissolução são discutidos neste trabalho.

$$
\begin{aligned}
& \mathrm{CuFeS}_{2}(\mathrm{~s})+4 \mathrm{Fe}^{3+}(\mathrm{aq}) \rightarrow \mathrm{Cu}^{2+}(\mathrm{aq})+ \\
& 5 \mathrm{Fe}^{2+}(\mathrm{aq})+2 \mathrm{~S}^{0}(\mathrm{~s})
\end{aligned}
$$




\section{I Efeito da Temperatura}

A análise da literatura mostra que a taxa de lixiviação da calcopirita é afetada pela temperatura [ 10$]$, sendo que a extração aumentou de $2 \%$ para $21 \%$ quando a temperatura cresceu de $50^{\circ} \mathrm{C}$ para $95^{\circ} \mathrm{C}$ no presente estudo (Figura 2), indicando um aumento expressivo na extração do cobre bem como a natureza bastante refratária da amostra mineral selecionada. Munoz et al. [ I I] também estudaram o efeito da temperatura na taxa de lixiviação da calcopirita com soluções ácidas de sulfato férrico, na faixa de $60^{\circ} \mathrm{C}$ a $90^{\circ} \mathrm{C}$ e descreveram uma relação direta entre as duas variáveis de acordo com uma função parabólica.

\subsection{Efeito da Concentração de Íon Férrico e do Potencial de Oxidação}

O efeito da concentração do oxidante (íon férrico) é apresentado na Figura 3. Pode-se observar que o aumento na concentração do oxidante de $0,01 \mathrm{~mol} / \mathrm{L}$ para $0,1 \mathrm{~mol} / \mathrm{L}$

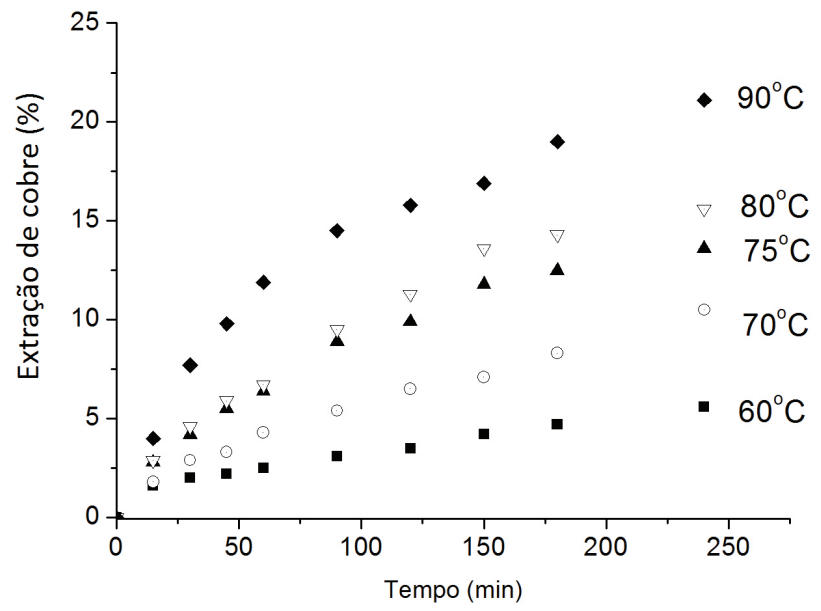

Figura 2. Efeito da temperatura na extração de cobre para lixiviação da amostra de calcopirita pulverizada e lavada com água ácida. Concentração de $0,5 \mathrm{~mol} / \mathrm{L}$ de $\mathrm{Fe}^{+3}$, I mol/L de ácido sulfúrico, I g/L de sólidos $(80 \%<20 \mu \mathrm{m})$ e agitação de $600 \mathrm{~min}^{-1}$.

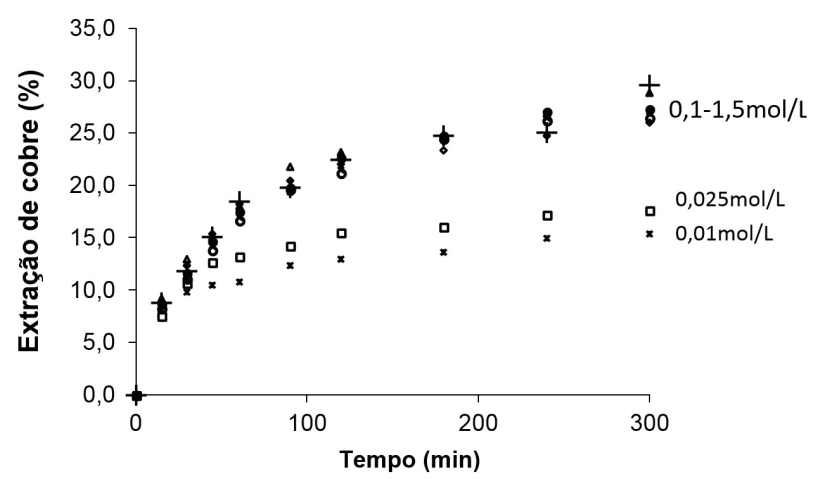

Figura 3. Efeito da concentração de $\mathrm{Fe}^{+3}$ na extração de cobre durante a lixiviação de uma amostra de calcopirita em meio sulfato. Concentração de $\mathrm{I} \mathrm{mol} / \mathrm{L}$ de $\mathrm{H}_{2} \mathrm{SO}_{4}, \mathrm{I} \mathrm{g} / \mathrm{L}$ de sólido $(80 \%-20 \mu \mathrm{m})$, agitação de $600 \mathrm{~min}^{-1}$ e $90^{\circ} \mathrm{C}$. aumentou o rendimento da extração de cobre de $15 \%$ para $26 \%$, após 5 horas de ensaio. Entretanto, não houve efeito na extração de cobre para as concentrações mais elevadas (até I,5 mol/L). Tais resultados são consistentes com outros trabalhos que não relataram nenhum efeito claro na cinética quando a concentração do íon $\mathrm{Fe}^{+3}$ excedeu $0,01 \mathrm{~mol} / \mathrm{L}[12,13]$.

Vários trabalhos indicam que a passivação da calcopirita é favorecida em elevados valores do potencial da solução $[3,14]$. Esses trabalhos indicam que existe um valor limite do potencial abaixo do qual a extração ocorre de maneira mais efetiva. No presente trabalho, o efeito do potencial de oxidação na extração de cobre foi avaliado em meio sulfato a partir da variação da relação $\mathrm{Fe}^{+3} / \mathrm{Fe}^{+2}$ mantendo-se a concentração total de ferro constante em $0,5 \mathrm{~mol} / \mathrm{L}$. Observou-se que o potencial de oxidação não variou devido à baixa concentração de sólidos. Dessa forma, somente os valores iniciais de Eh são apresentados na Figura 4.

Os resultados apresentados na Figura 4 indicam que a extração foi proporcional ao Eh, ou seja, a redução do Eh inicial diminuiu o rendimento da extração, ou seja, a extração de cobre reduziu-se de $16,4 \%$ para $6,1 \% \mathrm{com}$ a redução do Eh de $719,7 \mathrm{mV}$ para $350,3 \mathrm{mV}$ (vs $\mathrm{Ag} / \mathrm{AgCl})$. Em estudos de lixiviação de um concentrado contendo $80 \%$ de calcopirita em meio sulfato, Córdoba et al. [5] variaram a relação $\mathrm{Fe}^{+3} / \mathrm{Fe}^{+2}$ para obter potenciais de oxidação entre $300 \mathrm{mV}$ e $600 \mathrm{mV}$ (vs $\mathrm{Ag} / \mathrm{AgCl}$ ). Os autores concluíram que a redução do Eh de $600 \mathrm{mV}$ para $300 \mathrm{mV}$ aumentou a extração de cobre de $16 \%$ para $95 \%$, após treze dias de ensaio. Os autores sugeriram que o Eh controlava a nucleação e crescimento de uma camada de jarosita sobre os cristais de calcopirita o que seria a responsável pela passivação do mineral. Aparentemente, longos intervalos de tempo parecem ser necessários para mostrar o efeito de potenciais reduzidos no aumento da lixiviação da calcopirita, o que não pode ser observado no presente trabalho devido a curta extensão dos ensaios realizados.

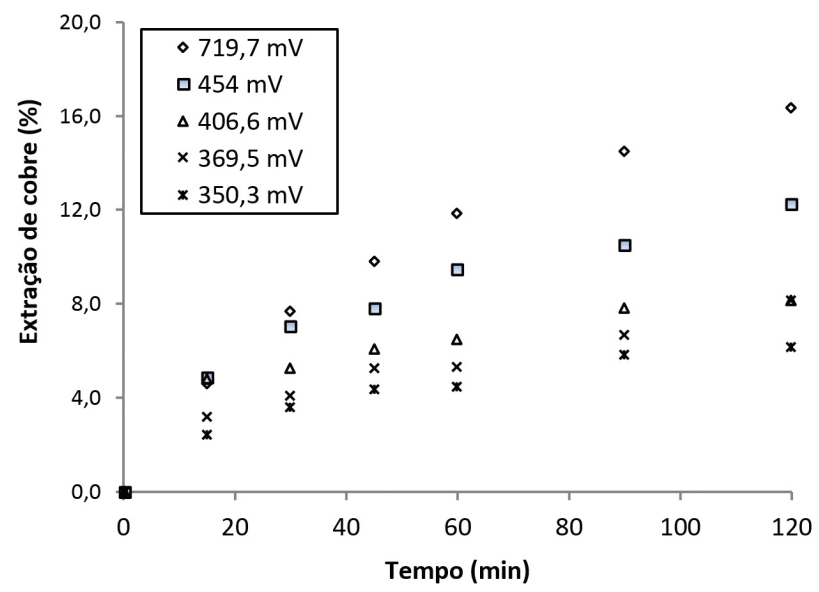

Figura 4. Efeito do Eh na extração de cobre durante a lixiviação da amostra de calcopirita. Condições experimentais: I ol/L $\mathrm{H}_{2} \mathrm{SO}_{4}, 0,5$ $\mathrm{mol} / \mathrm{L}$ de ferro total, I $\mathrm{g} / \mathrm{L}$ sólidos $(80 \%<20 \mu \mathrm{m}), 600 \mathrm{~min}^{-1}$ e $90^{\circ} \mathrm{C}$. 


\subsection{Análise Cinética}

A análise cinética utilizou a estequiometria da reação de lixiviação mostrada na Equação I e envolveu apenas a determinação da energia de ativação. A ordem de reação em relação ao agente oxidante não foi determinada uma vez que o íon $\mathrm{Fe}^{3+}$ teve pequeno efeito na cinética de lixiviação da calcopirita.

O modelo do núcleo não reagido foi aplicado aos dados experimentais considerando concentração dos reagentes e temperatura constante; partículas de geometria esférica (Figura 5) e que a difusão no filme não controlava a lixiviação de cobre para a velocidade de agitação selecionada.

Com base nas premissas adotadas, a etapa controladora do processo de lixiviação poderia ser reação química ou a difusão na camada de produto. Supondo que o controle fosse por reação química, a Equação 2 descreveria a cinética do processo, onde $\alpha$ é a fração reagida.

$$
\mathrm{I}-(\mathrm{I}-\alpha)^{1 / 3}=\mathrm{K}_{\mathrm{R} . \mathrm{t}}, \mathrm{K}_{\mathrm{R}}=\frac{\mathrm{I} \cdot \mathrm{K} \cdot\left[\mathrm{Fe}^{3+}\right]^{\mathrm{n}}}{4 \cdot \rho_{\text {solido }} \cdot \mathrm{r}_{\mathrm{o}}}
$$

Caso a etapa controladora fosse difusão na camada de cinzas, a Equação 3 representaria a cinética de lixiviação do sulfeto pelo ín $\mathrm{Fe}^{3+}$ :

$$
\mathrm{I}-3(\mathrm{I}-\alpha)^{2 / 3}+2(\mathrm{I}-\alpha)=\mathrm{K}_{\mathrm{d} . \mathrm{t}} \mathrm{K}_{\mathrm{D}}=\frac{6 \cdot \mathrm{D}_{\mathrm{eff}} \cdot\left[\mathrm{Fe}^{3+}\right]}{4 \cdot \rho_{\text {solido }} \cdot \mathrm{r}_{\mathrm{o}}{ }^{2}}
$$

Nas Equações 2 e 3, $K$ é a constante de velocidade; $n$ a ordem de reação em relação ao íon $\mathrm{Fe}^{3+} ; D_{\text {eff }}$ o coeficiente de difusão efetivo; $\rho_{\text {sólido }}$ é a densidade molar do sulfeto e $r_{0}$, $\circ$ tamanho de partícula em $\mathrm{t}=0$.

Levenspiel [15] argumenta que o SCM foi desenvolvido orginalmente para reações gás/sólido e a hipótese do estado quasi-estacionário (usada no desenvolvimento da Equação 3) foi proposta considerando que a velocidade de movimento da interface entre núcleo não reagido e a camada de cinza era muito mais lenta do que a velocidade de difusão do reagente até interface. Em sistemas gás/sólido, tal velocidade é cerca de 1.000 vezes menor do que a velocidade de transporte do gás, através da camada de cinzas. Entretanto, para sistemas sólido/líquido, Levenspiel [15] cita que o valor da razão de velocidades entre $\mathrm{o}$ avanço da interface e $\mathrm{o}$ transporte de reagente através da camada de cinzas é próximo de I, o que questionaria a validade da hipótese do estado quasiestacionário. Dessa forma, a aplicação do modelo do núcleo não reagido em sistemas sólido/líquido de interesse para a hidrometalurgia requereu uma abordagem específica.

Nesse sentido, uma revisão sobre o uso da aproximação do estado quasi-estacionário em sistemas hidrometalúrgicos foi conduzida por Liddell [16] que sugere dois critérios simples para definir se essa aproximação é válida. O primeiro deles $(\delta)$ foi proposto originalmente por Bischoff [ 17$]$, sendo que o erro associado com a aproximação do estado quasiestacionário é pequeno para $\delta<<$ I (Equação 4):

$$
\delta=\frac{C_{A}}{\rho_{\text {solido }}}
$$

O segundo critério foi proposto por Taylor et al. [18], sendo a aproximação do estado quasi-estacionário válida para $\gamma_{1} \leq 0.1$ (Equação 5):

$$
\gamma_{1}=\frac{\mathrm{aC}_{\mathrm{A}}}{\mathrm{b} \rho_{\text {solido }}}
$$

Onde $C_{A}$ é a concentração do reagente na fase aquosa, e $a$ e $b$ são os coeficientes estequiométricos para o reagente sólido e aquoso, respectivamente. Considerando-se a equação global da oxidação da calcopirita por íons $\mathrm{Fe}^{+3}$ em meio ácido e uma concentração de íon férrico igual a $0,5 \mathrm{~mol} / \mathrm{L}$, obteve-se $\delta=0,022$ e $\mathrm{y}_{1}=5,46 \times 10^{-3}$, nas condições do presente trabalho. Segundo Liddell [16], estes valores atendem a aproximação de estado quasi-estacionário e justificam a aplicação do modelo do núcleo não reagido aos dados experimentais.

No caso de controle por reação química, o gráfico de $\mathrm{I}-(\mathrm{I}-\alpha)^{1 / 3}$ versus tempo é uma linha reta com inclinação $\mathrm{K}_{\mathrm{R}}$.
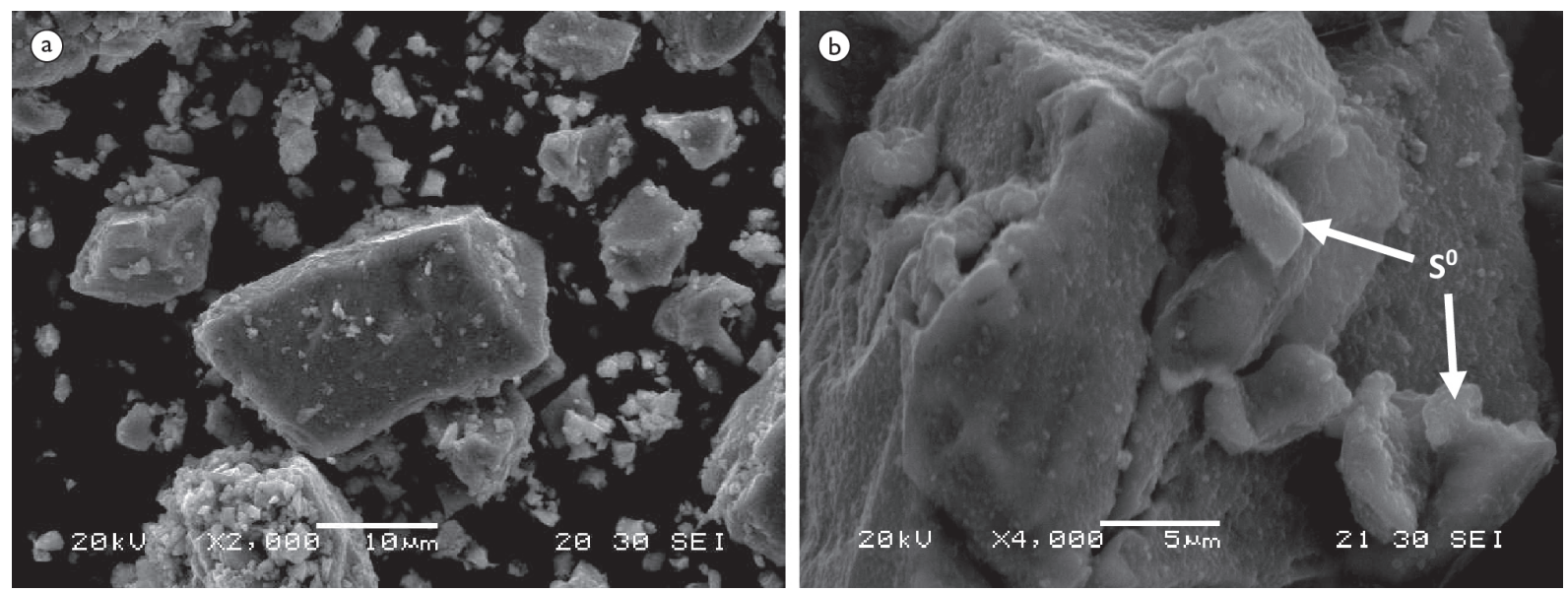

Figura 5. Análise por MEV-EDS da amostra inicial (a) e; do resíduo de lixiviação (b). 
Entretanto, os valores experimentais de $\mathrm{I}-(\mathrm{I}-\alpha)^{1 / 3}$ versus tempo não se aproximaram de uma linha reta (baixos valores do coeficiente de correlação) o que sugeriu que o controle do processo de lixiviação da calcopirita em meio sulfato com íon férrico não era controlado por reação química, apesar do grande efeito da temperatura na extração de cobre (Figura 2).

Para o controle por difusão na camada de cinzas, a Figura 6 mostra que o ajuste de $I-3(I-\alpha)^{2 / 3}+2(I-\alpha)$ versus tempo se aproximou muito de uma linha reta, obtendo-se um ajuste $\left(r^{2}\right)$, superior a 0,96 , para a maioria dos experimentos. Esse fato sugeriu que a cinética de lixiviação poderia ser descrita pelo modelo do núcleo não regido com controle por difusão na camada de cinza.
A partir dos valores da constante de velocidade $\left(K_{D}\right)$ obtidos pelo ajuste ao controle por difusão na camada de cinza (Equação 3), foi calculada a energia de ativação do processo usando a equação de Arrenhius (Figura 7), sendo o valor calculado igual a $103,9 \pm 6,5 \mathrm{~kJ} / \mathrm{mol}$. Dutrizac et al. [12] estudaram a cinética de dissolução de um concentrado calcopirítico com íon férrico em meio sulfato no intervalo de temperatura entre $50^{\circ} \mathrm{C}$ e $94^{\circ} \mathrm{C}$ e determinaram uma energia de ativação igual a $7 \mathrm{l} \mathrm{kJ} / \mathrm{mol}$. Da mesma forma, Munoz et al. [I I] encontraram energia de ativação igual a $84 \mathrm{~kJ} / \mathrm{mol}$ para dissolução de um concentrado calcopirítico em sulfato férrico para temperaturas entre $60^{\circ} \mathrm{C}$ e $90^{\circ} \mathrm{C}$.

Os processos difusionais não são muito afetados pela temperatura e não se espera valores de energia de ativação

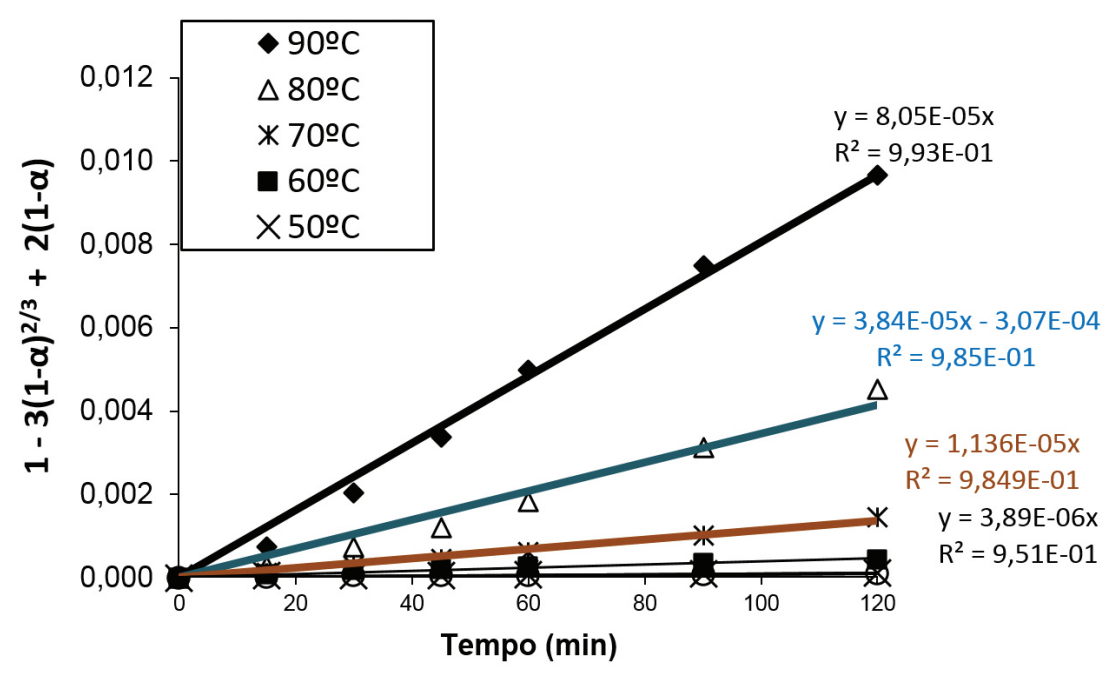

Figura 6. Ajuste dos dados experimentais ao modelo do núcleo não reagido com controle por difusão na camada de cinzas. Temperatura entre $50^{\circ} \mathrm{C}$ e $90^{\circ} \mathrm{C} ; 0,5 \mathrm{~mol} / \mathrm{L}$ de $\mathrm{Fe}^{+3} ; \mathrm{I} \mathrm{mol} / \mathrm{L} \mathrm{de} \mathrm{H}_{2} \mathrm{SO}_{4} ; \mathrm{I} \mathrm{g} / \mathrm{L}$ de sólidos $(80 \%<20 \mu \mathrm{m}) ; 600 \mathrm{~min}^{-1}$.

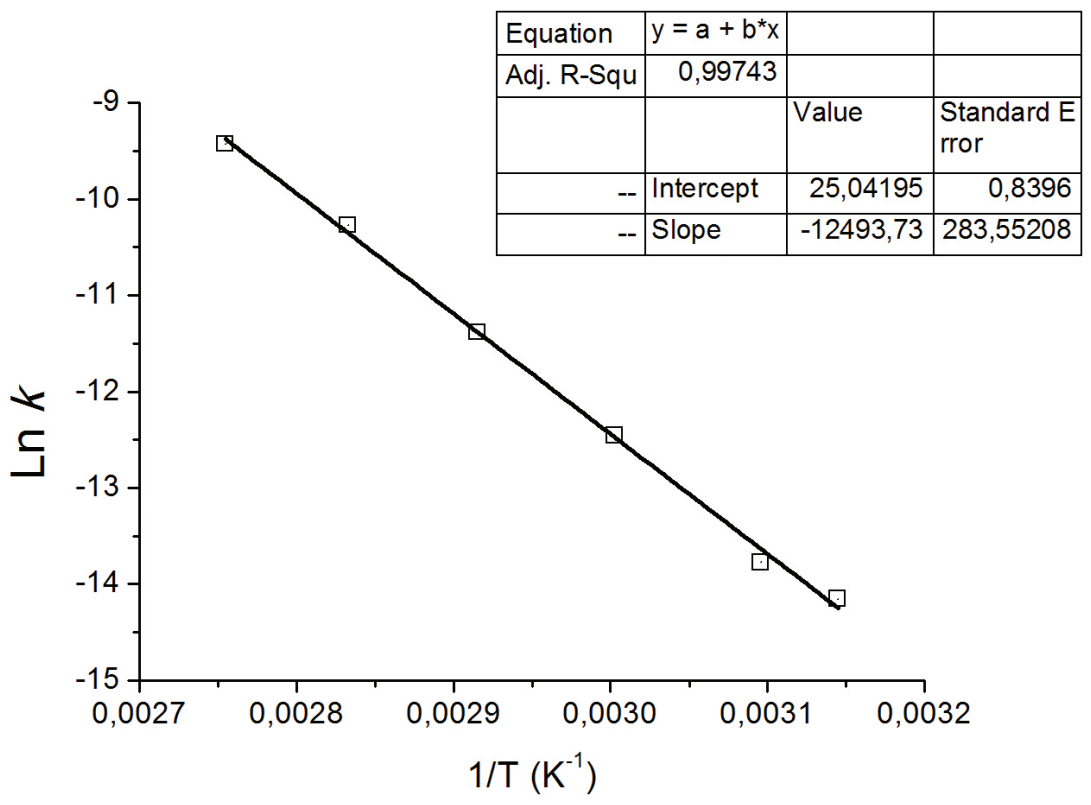

Figura 7. Gráfico de Arrhenius para a lixiviação da calcopirtia por Fe ${ }^{3+}$ para $0,5 \mathrm{~mol} / \mathrm{L} \mathrm{Fe}^{+3} ; \mathrm{I} \mathrm{mol} / \mathrm{L} \mathrm{de}_{2} \mathrm{SO}_{4} ; \mathrm{I} g / \mathrm{L}$ de sólidos $(80 \%<20 \mu \mathrm{m})$ e $600 \mathrm{~min}^{-1}$. 
muito superiores a $20 \mathrm{~kJ} / \mathrm{mol}$ para controle por difusão na camada de cinza. No presente estudo, a distribuição do tamanho de partícula pode ter afetado o ajuste das Equações 2 e 3 aos dados experimentais. Isso ocorre por que a formulação original do SCM é para partículas de mesmo tamanho e Gbor e jia [6] demostraram que no caso de controle químico, a distribuição de tamanho das partículas pode afetar a extração de maneira tal que, além de não se observar o ajuste à Equação 2 (o que seria esperado), os dados de extração podem se ajustar à Equação 3 (controle por difusão na camada de cinza). Segundo Gbor e Jia [6], a não consideração do efeito do tamanho de partículas no desenvolvimento da Equação 2 ocorre para valores do coeficiente de variação $(\mathrm{CV})$ da distribuição de tamanho das partículas entre 0,7 e I,2 [6]. No presente estudo, o elevado valor da energia de ativação sugere controle químico e a distribuição de tamanho de partículas estudadas tinha um valor de CV igual a 0,78 , ou seja, dentro da faixa onde observa-se o ajuste a equação para a controle na camada de cinza, mesmo sendo o processo controlado quimicamente, segundo Gbor e Jia [6]. Trabalhos futuros abordarão a aplicação do modelo proposto por Gbor e jia [6] aos dados experimentais obtidos.
A amostra de calcopirita estudada apresentou-se bastante refratária ao ataque pelo íon $\mathrm{Fe}^{3+}$ em meio sulfato com extrações máximas em torno de $25 \%$. A temperatura exerceu forte influência sobre a extração de cobre, pois sua elevação (de $50^{\circ} \mathrm{C}$ para $90^{\circ} \mathrm{C}$ ) aumentou a extração de $5 \%$ para $21 \%$. Por outro lado, o aumento da concentração de íon férrico favoreceu uma maior dissolução de cobre apenas para concentrações menores que $0, \mathrm{I} \mathrm{mol} / \mathrm{L}$. Nessa condição, o rendimento da extração de cobre foi de $26 \%$, após 5 horas de ensaio.

A modelagem cinética foi feita utilizando o SCM e indicou que a etapa controladora da reação é a difusão na camada de produto apesar do elevado valor observado para a energia de ativação $(103,9 \pm 6,5 \mathrm{~kJ} / \mathrm{mol})$. Tal resultado é devido ao efeito da distribuição de tamanho de partículas (PSD) na análise cinética. Como o SCM foi desenvolvido para partículas do mesmo tamanho e a PSD da amostra estudada tinha um coeficiente de correlação igual a 0,78 , observou-se o ajuste à equação que descreve o controle na camada de cinza mesmo sendo o processo controlado quimicamente, como sugerido pelo valor da energia de ativação.

\section{CONCLUSÕES}

\section{Agradecimentos}

Os autores agradecem à CAPES, ao CNPq, à FAPEMIG e à FINEP pelo apoio. As bolsas de iniciação científica (CNPq), mestrado (CAPES) e produtividade em pesquisa (CNPq) são especialmente reconhecidas.

\section{REFERÊNCIAS}

I Ciminelli VST. Hidrometalurgia. In: Fernandes FRC, Matos GMM, Castilhos ZC, Luz AB, editors. Tendências Tecnológicas Brasil 2015. Rio de Janeiro: CETEM; 2005. p. I57-I74.

2 Cruz FLS, Oliveira VA, Guimarães D, Souza AD, Leão VA. High-temperature bioleaching of nickel sulfides: Thermodynamic and kinetic implications. Hydrometallurgy. 2010;105(I-2): 103-109. http://dx.doi.org/10.1016/j. hydromet.2010.08.006.

3 Córdoba EM, Muñoz JA, Blázquez ML, González F, Ballester A. Leaching of chalcopyrite with ferric ion. Part I: General aspects. Hydrometallurgy. 2008;93(3-4):8I-87. http://dx.doi.org/10.1016/j.hydromet.2008.04.015.

4 Hackl RP, Dreisinger DB, Peters E, King JA. Passivation of chalcopyrite during oxidative leaching in sulfate media. Hydrometallurgy. 1995;39(I-3):25-48. http://dx.doi.org/10.1016/0304-386X(95)00023-A.

5 Córdoba EM, Muñoz JA, Blázquez ML, González F, Ballester A. Leaching of chalcopyrite with ferric ion. Part II: Effect of redox potential. Hydrometallurgy. 2008;93(3-4):88-96. http://dx.doi.org/10.1016/j.hydromet.2008.04.016.

6 Gbor PK, Jia CQ. Critical evaluation of coupling particle size distribution with the shrinking core model. Chemical Engineering Science. 2004;59(I0): 1979-1987. http://dx.doi.org/I0. I0I6/j.ces.2004.0I.047.

7 Souza AD, Pina PS, Leão VA, Silva CA, Siqueira PF. The leaching kinetics of a zinc sulphide concentrate in acid ferric sulphate. Hydrometallurgy. 2007;89(I-2):72-8I. http://dx.doi.org/I0.10 I6/j.hydromet.2007.05.008.

8 Santos FMF, Pina PS, Porcaro R, Oliveira VA, Silva CA, Leão VA. The kinetics of zinc silicate leaching in sodium hydroxide. Hydrometallurgy. 2010; I02(I-4):43-49. http://dx.doi.org/I0. I016/j.hydromet.2010.01.010.

9 Souza AD, Pina PS, Lima EVO, Silva CA, Leão VA. Kinetics of sulphuric acid leaching of a zinc silicate calcine. Hydrometallurgy. 2007;89(3-4):337-345. http://dx.doi.org/10.1016/j.hydromet.2007.08.005. 
10 Carneiro MFC, Leão VA. The role of sodium chloride on surface properties of chalcopyrite leached with ferric sulphate. Hydrometallurgy. 2007;87(3-4):73-82. http://dx.doi.org/I0.1016/j.hydromet.2007.01.005.

II Munoz PB, Miller JD, Wadsworth ME. Reaction mechanism for the acid ferric sulfate leaching of chalcopyrite. MTB. 1979; I0(2): I49-I58. http://dx.doi.org/10.1007/BF02652458.

12 Dutrizac JE, MacDonald RJC, Ingraham TR. The kinetics of dissolution of synthetic chalcopyrite in aqueous acidic ferric sulfate solutions. Transactions of the Metallurgical Society of AIME. 1969;245:955-959.

13 Munoz PB, Miller JD, Wadsworth ME. Reaction mechanism for the acid ferric sulfate leaching of chalcopyrite. MTB. 1979; I0(2): I49-I58. http://dx.doi.org/I0.1007/BF02652458. [English.]

14 Hiroyoshi N, Miki H, Hirajima T, Tsunekawa M. Enhancement of chalcopyrite leaching by ferrous ions in acidic ferric sulfate solutions. Hydrometallurgy. 200 I;60(3): I85-197. http://dx.doi.org/10.1016/S0304-386X(00)00I55-9.

15 Levenspiel O. Chemical reaction engineering. New York: John Wiley \& Sons; 1962.578 p.

16 Liddell KC. Shrinking core models in hydrometallurgy: What students are not being told about the pseudo-steady approximation. Hydrometallurgy. 2005;79(I-2):62-68. http://dx.doi.org/10.1016/j.hydromet.2003.07.0II .

17 Bischoff KB. Further comments on the pseudo steady state approximation for moving boundary diffusion problems. Chemical Engineering Science. 1965;20(8):783-784. http://dx.doi.org/10.1016/0009-2509(65)80065-3.

18 Taylor PR, Matos M, Martins GP. Modeling of noncatalytic fluid-solid reactions: The quasi-steady state assumption. MTB. 1983; I4(I):49-53. http://dx.doi.org/10.1007/BF02670868.

Recebido em: 13 Ago. 2014

Aceito em: 05 Jan. 2015 